\title{
Human Mammary Tumor Virus (HMTV) sequences in human milk
}

Teiko Nartey ${ }^{1}$, Heberth Moran ${ }^{1}$, Tania Marin ${ }^{1}$, Kathleen F Arcaro ${ }^{2}$, Douglas L Anderton ${ }^{3}$, Polly Etkind ${ }^{1}$, James F Holland', Stella M Melana ${ }^{1 *}$ and Beatriz G-T Pogo ${ }^{1,4^{*}}$

\begin{abstract}
Background: Retroviral sequences $90-95 \%$ homologous to the mouse mammary tumor virus (MMTV) were present in 38\% of the breast cancers studied from American women and were not detectable in non-tumor breast tissue from the same patient. The entire proviral structure was described and viral particles were isolated from primary cultures of human breast cancer. This virus was designated as human mammary tumor virus (HMTV). Hormone response elements present in the HMTV Long-Terminal-Repeat (LTR) suggest a mechanism for association of HMTV with hormonally responding tissues. In fact, the incidence of HMTV sequences is higher in gestational breast cancers, which are associated with hormonal changes. Milk epithelial cells are also under hormonal regulation and therefore are excellent specimens for HMTV sequence detection.
\end{abstract}

Methods: The HMTV sequence was studied in milk samples from lactating women recruited with increased risk of breast cancer because they had undergone breast biopsies (Biopsy-Group) and lactating women without breast biopsies (Reference-Group).

Results: HMTV-env sequences were detected by PCR in milk of $7.61 \%$ of 92 women of the Reference-Group and in $20.55 \%$ of 73 women of the Biopsy-Group (p: 0.015). The sequences were 94-98\% homologous to MMTV. HMTV-env and HMTV-env/LTR junction sequences were detected in high-speed pellet RNA, implying the presence of HMTV viral particles. PCR assays to detect the murine mitochondrial cytochrome oxidase gene and intracisternal-A-type particle sequences were performed to rule out mouse mitochondrial or genomic DNA contamination. Eight women of the 73 Biopsy-Group participants had breast cancer and the milk of only one of these eight women had HMTV-env sequences. In the remaining 65 women of the Biopsy-Group, under enough clinical suspicion to lead to biopsy, HMTV was detected in 14, nearly three times the number of milks as compared to the Reference-Group (21.54\% versus $7.61 \%$; p: 0.016)

Conclusion: The significance of HMTV in milk from the Reference-Group, the greater frequency in the milk of women who had undergone a breast biopsy and its possible infectivity for infants are important questions under study. The similarity of HMTV to MMTV is striking and suggests one possible avenue for viral transmission in humans.

Keywords: Retrovirus, Breast cancer, HMTV, Milk, Breast biopsies

\footnotetext{
* Correspondence: stella.melana@mssm.edu; beatriz.pogo@mssm.edu

${ }^{1}$ The Tisch Cancer Institute and Department of Medicine, Icahn School of Medicine at Mount Sinai, 1 Gustave L. Levy Place, New York, NY 10029, USA ${ }^{4}$ Department of Microbiology, Icahn School of Medicine at Mount Sinai, 1 Gustave L. Levy Place, New York, NY 10029, USA

Full list of author information is available at the end of the article:
} 


\section{Background}

The presence of viral particles with the morphological characteristics of betaretroviruses was first described in human milk by Feller and Chopra [1,2]. In 1971 Moore et al. [3] also described a betaretrovirus similar to mouse mammary tumor virus (MMTV) in the milk from $5 \%$ of American women with no familial history of breast cancer, $60 \%$ of American women with familial breast cancer and $39 \%$ from Parsi women [3], who have a three-fold greater risk of developing breast cancer than the nonParsi population in Bombay [3]. Schlom et al. (1972) [4] found Reverse Transcriptase (RT) activity and RNA in viral particles of the same density as that of betaretroviruses in human milk. A human protein related to the envelope protein (Env) of MMTV was identified from a pool of human milk samples from 300 healthy women [5]. Furthermore, another retroviral protein, p27, related to MMTV gag gene was also shown to be present in some established breast cancer cell lines and in cells from human milk $[6,7]$. When human endogenous retroviruses (HERV) with homology to MMTV were described in the 1980's, it was assumed that the particles described above were endogenous in origin [8]. However, the presence of several other viruses in milk has now been studied $[9,10]$ and a recent report indicated that MMTV-like sequences were present in DNA of milk cells of $5 \%$ of healthy Australian Women [11].

We have reported previously the presence of sequences from a retrovirus $90-95 \%$ homologous to MMTV in 38$40 \%$ of the breast cancers studied in American women [12], whereas these sequences are not detectable in nontumor mammary tissue from the same breast that contained the tumor [13]. The whole proviral structure has been described and designated as human mammary tumor virus (HMTV) [14]. The presence in human breast cancer of sequences homologous to MMTV has been confirmed by several groups [15-19]. Other authors have designated these sequences as MMTV-like, and thus appear to be the same virus as HMTV [15-19].

HMTV retroviral particles have been isolated from primary cultures of human metastatic breast cancer cells [20] and expression of HMTV proteins has been shown in cells containing HMTV proviral DNA sequences [21]. We have also found that the incidence of HMTV sequences is higher in inflammatory [22] and gestational breast cancers than in sporadic specimens [23]. Gestational breast cancers, by definition, are associated with major hormonal changes and hormone responsive elements present in the LTR of HMTV [23], together suggest a molecular mechanism to explain viral association with hormonally responding tissues. Epithelial cells from milk, because of their association with hormonal changes, are excellent specimens in which to search for HMTV sequences.
In this communication we show that HMTV-env sequences are present in the DNA of milk cells from $20.55 \%$ of the women who had previously undergone breast biopsies as compared to only $7.61 \%$ of the women in the Reference-Group. In addition, the detection of HMTV-env/LTR junction sequences from the highspeed pellet RNA indicates the presence of HMTV viral particles in several milk samples of Biopsy and Reference-Group women.

\section{Results and discussion}

Milk samples from 97 women in the Reference-Group and 79 women in the Biopsy-Group were analyzed. The $\beta$-globin gene could be amplified in milk samples from 92 of the 97 women in the Reference-Group and 74 of the 79 women in the Biopsy-Group and thus they were qualified for further analysis. All the DNA samples showing presence of viral sequences were tested for the presence of murine mitochondrial cytochrome oxidase gene (MoMt) and murine intracisternal A-particles (IAP) by PCR. Only one sample in the Biopsy-Group was found to contain murine DNA and was eliminated from future study.

The results (Table 1) indicated that HMTV-env was detected in the DNA of 7 of 92 (7.61\%) Reference-Group women. For the Biopsy-Group, 15 of the 73 women (20.55\%) had HMTV sequences in the DNA of their milk cells. This difference between both groups was statistically significant (p: 0.015). The higher percentage of women with HMTV sequences in their breast milk DNA was not correlated with any of the demographic parameters previously examined including age at time of milk donation, time after delivery, number of live births, or age at first pregnancy [24,25]. Eight women in the Biopsy-Group were diagnosed with breast cancer and one of them was found to have HMTV sequences in her milk cell DNA. Fourteen of the 65 (21.54\%) biopsied women without breast cancer had HMTV-env sequences in their milk. One representative experiment is shown in Figure 1.

When HMTV-env amplicon sequences were compared to MMTV (GenBank: M15122) sequences reported in the GenBank they were found to be 94 to $98 \%$ homologous. No major differences were found between HMTV sequences isolated from the women in either group. The HMTV-env sequence was submitted to the GenBank.

HMTV particle sequences were also sought in the high-speed pellet (P2) RNA from milk of the same

Table 1 Presence of HMTV-env sequences in human milk genomic DNA (P1)

\begin{tabular}{lcccc}
\hline Group & Number of women & env sequences & $\%$ & $\mathbf{P}^{*}$ \\
\hline Reference & 92 & 7 & 7.61 & - \\
Biopsied & 73 & 15 & 20.55 & 0.015
\end{tabular}

*The chi-square test was used to compare the two groups. 


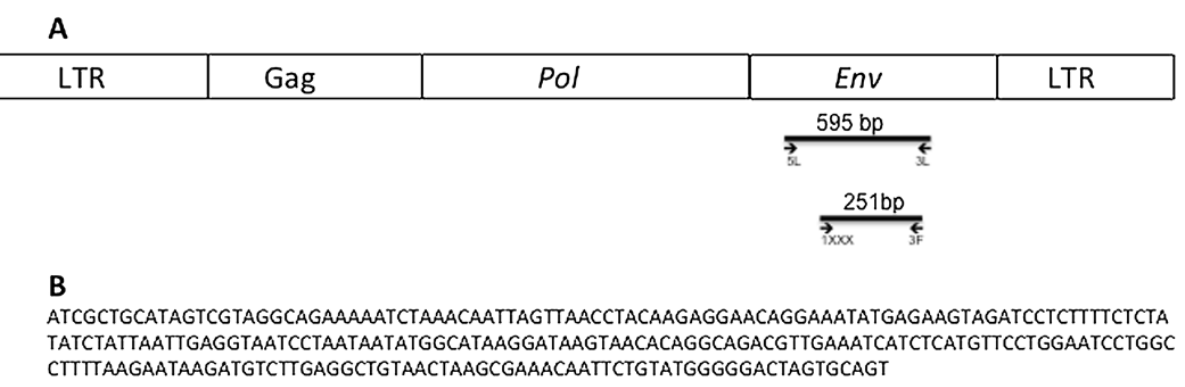

C

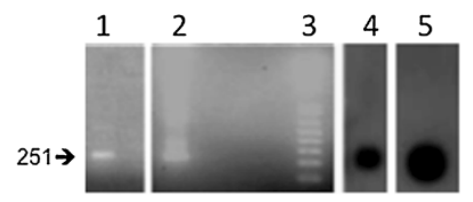

Figure 1 Detection of HMTV-env gene sequences in milk cells. PCR was performed using primers $5 L$ and $3 L$ and nested PCR using primers $1 X X X$ and $3 \mathrm{~F}$ as described in Methods. A. Diagram of HMTV proviral DNA structure and location of the primers. B. HMTV-env DNA sequence using primers 1XXX and 3 F in a nested PCR reaction (GenBank KJ831809). C. 2\% agarose gel electrophoresis of HMTV-env 251 bp amplicon. Lane 1: HMTV-env positive sample, Lane 2: HMTV-env positive control, Lane 3: 1 kb plus DNA ladder. Southern blot hybridization using ${ }^{32}$ P labeled probe as described in Methods. Lane 4: HMTV-env positive sample. Lane 5: HMTV-env positive DNA control.

Biopsy and Reference Group women whose milk cell DNA had HMTV sequences. RNA was extracted as described in Methods and RT-PCR carried out for HMTV-env (Figure 2) and HMTV-env/LTR junction sequences (Figure 3). The HMTV sequences from the cDNA were compared to sequences present in the GenBank. HMTV-env sequences were 94 to $98 \%$ homologous to MMTV (GenBank: M15122) and HMTV-env/LTR junction sequence was 96\% homologous to MMTV (GenBank: M15122). Results in Table 2 indicate that HMTV-env and HMTV-env/LTR junction sequences were amplified from P2 RNA when the HMTV-env also was detected in the genomic DNAs from P1. HMTV-env sequences were amplified in P2 RNA from 2 out of 3 women of the Reference-Group. All three of these women also had HMTV sequences detected in their genomic DNA and HMTV-env/LTR junction sequences

A

\begin{tabular}{|c|c|c|c|}
\hline Gag & $\mathrm{Pol}$ & Env & LTR \\
\hline
\end{tabular}

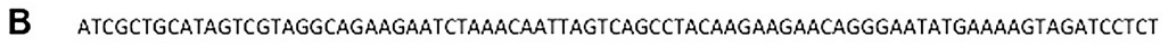
CTICTCTATATCTATTAGCTGAGGTAATCCTAATAATATGGCATAAGGGTAAGTAACACAGGCAGATGTAGGAATCATCTCATGT TCTTGAAATCCTGGCTITITGAGAATAAGATGTCTTGAGGCTGCAACTAAGCGAAACAATTTTGTATGGGGGACTAGTGCAT

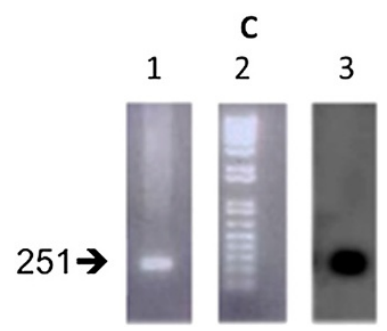

Figure 2 Detection of HMTV-env gene sequences in P2 RNA. CDNA synthesized from high speed pellet (P2) RNA using polydT as primer, PCR was performed using primers $5 L$ and $3 L$ and nested $P C R$ using primers $1 X X X$ and $3 F$ as described in Methods. A. Diagram of HMTV viral RNA structure and location of the primers. B. HMTV-env CDNA sequence was amplified using primers 1 XXX and $3 \mathrm{~F}$ in a nested PCR reaction (GenBank KJ831810). C. 2\% agarose gel electrophoresis of HMTV-env 251 bp amplicon. Lane 1: HMTV-env positive sample. Lane 2: 1 kb plus DNA ladder. Lane 3: Southern blot hybridization using ${ }^{32} \mathrm{P}$ end labeled probe for HMTV-env positive sample. 
present in P2 RNA. In the case of the Biopsied-Group, HMTV-env and HMTV env/LTR junction sequences were amplified in P2 RNA from 4 out of 5 women whose genomic DNA contained HMTV sequences. HMTV-env/LTR junction sequences were detected in a total of 7 P2 RNA samples of which one contained enough material to allow sequencing. HMTV-env and env/LTR junction sequences from P2 RNA have been submitted to the GenBank. Absence of glycerol-3-phosphate dehydrogenase gene (G3DPH) mRNA from high-speed pellets and the presence of HMTV-env/LTR junction sequences is consistent with the presence of virion RNA.

During lactation, the mammary gland undergoes molecular changes in response to hormonal influences. Gestational breast cancer that develops during pregnancy or lactation is considered to be very aggressive and related to hormonal changes, and it has been shown to have a high incidence of viral HMTV sequences [23].

In this communication evidence is presented that milk cells of $7.61 \%$ of women from a Reference-Group contain HMTV viral sequences in their genomic DNA. The percentage of women with HMTV sequences in the milk cells from the Biopsy-Group is remarkably higher (20.55\%). The usual indications for biopsy are physical, radiologic, or sonographic findings, which raise the possibility of breast cancer [26]. According to the American Cancer Society, roughly one out of five breast biopsies results in a diagnosis of cancer [26]. Eight of the women in the Biopsy-Group were found to have breast cancer and one of the eight had HMTV sequences in her milk. Of the remaining 65 women of the Biopsy-Group, under enough clinical suspicion to lead to biopsy, 21.54\% contain HMTV sequences in their milks. This percentage represents a nearly three-fold increase compared to the ReferenceGroup women (p: 0.016). This finding suggests that the presence of the HMTV sequences is associated with increased breast cancer risk.

The present results confirm, extend, and validate the findings of the 1960s and 1970s that provided morphological and immunologic evidence of viral particles similar to MMTV in human milk [1-4]. These results are in agreement with the observations of Johal et al. [11] that env sequences are present in the milks from $5 \%$ of Australian healthy women. We have proven that the sequences are not derived from murine contamination, and add the striking observation that clinical findings that led to a biopsy were associated with a nearly 3-fold increase in women who had HMTV sequences in their milk. Furthermore, we

Table 2 Detection of HMTV sequences in human milk high speed Pellet P2 RNA

\begin{tabular}{lccc}
\hline Group & HMTV-env sequences in genomic DNA (P1) & HMTV-env sequences in RNA (P2) & HMTV-env/LTR junction sequences in RNA (P2) \\
\hline Reference & 3 & $2 / 3$ \\
Biopsied & 5 & $4 / 5$ & $4 / 3$ \\
\hline
\end{tabular}


have also shown that there is HMTV-env/LTR junction sequences in the high-speed pellet RNA from milk supernatants, which is consistent with the presence of viral particles.

\section{Conclusion}

The significance of HMTV in human milk, its greater frequency in the milk of women who have undergone a breast biopsy and its possible transmission to their infants, are important questions still under study. The similarity of HMTV and MMTV in this regard is striking and suggests one possible avenue for human transmission.

Breastfeeding in general has been considered to lower the risk of eventual breast cancer in the offspring [27]. However, nearly all infants have been exposed to colostrum, even when mothers deny having breastfed their children $[11,28,29]$. Therefore, a small proportion of breastfed infants who might have been at increased risk may have been hidden in the data. The results presented here pave the way to stimulate research in this area and to develop strategies that are precisely targeted to prevent transmission by HMTV carrying women, without eliminating the well-known benefits of breastfeeding for those who do not carry the virus. Such attempts have been very successful in Japan, where HTLV, the causative agent of adult T-cell leukemia, has been significantly reduced in the population by measures that dissuade HTLV carrier mothers from breastfeeding [30].

\section{Methods}

\section{Milk sample collection}

Milk samples were provided by K. F. Arcaro at the University of Massachusetts at Amherst, MA. Collection was performed with the women's consent, following the conditions approved by the institutional review board (IRB) of the University of Massachusetts-Amherst as described in previous publications $[24,25]$. Milk from two groups of American women was studied. One group, referred to as the Reference-Group, involved nursing women without biopsies. The other group, referred to as the Biopsy-Group, consisted of nursing women from across the US who either had undergone a breast biopsy at some point in their lives or were scheduled to have a breast biopsy. The Biopsy-Group represents women whose clinical or radiological findings suggested the need for a biopsy to exclude breast cancer and are thus considered to be at increased risk of breast cancer [25]. Frozen milks were sent on dry ice to B. G-T Pogo. For each of 97 women in the Reference-Group, a single sample ( 80 to $120 \mathrm{ml}$ ) of milk was received. For 60 women in the Biopsy-Group matched samples (80 to $120 \mathrm{ml}$ ) of milk from the left and right breasts were received along with a single sample of similar volume from either the left or right breasts of an additional 19 women. Thus a total of 139 samples from 79 women was received from the Biopsy-Group. Milks were kept frozen at $-20^{\circ} \mathrm{C}$ until processing. The milk samples were first centrifuged at $1,000 \times \mathrm{g}$ for 30 minutes at $4^{\circ} \mathrm{C}$. The fat layer was removed and supernatant designated S1 transferred to a new tube and the resulting cell pellet (P1) was re-suspended in PBS and DNA was extracted. The supernatant was then centrifuged at $10,000 \times \mathrm{g}$ for $30 \mathrm{~min}$. and the resulting supernatant was centrifuged at $100,000 \times \mathrm{g}$ for $150 \mathrm{~min}$ at $4^{\circ} \mathrm{C}$. This high-speed pellet designated as $\mathrm{P} 2$ was stored at $-80^{\circ} \mathrm{C}$.

\section{DNA extraction}

DNA from P1 was extracted according to the manufacturer's instructions (Life Technology Inc., Carlsbad, CA). The suitability of DNAs for PCR was tested by amplifying a 250 bp sequence of the $\beta$-globin gene using primers $\mathrm{GH}$ 20 (5' GAAGAGCCAAGGACAGGTAC-3') and PCO4 (5' CAACTTCATCCACGTTCACC-3') under the conditions previously described [31]. $\beta$-globin sequences were amplified in all reported specimens.

\section{Detection of HMTV-env sequences}

PCRs were performed on $250 \mathrm{ng}$ of DNA using Illustra PureTaq Ready-To-Go PCR beads (GE Healthcare Inc., Piscataway, NJ) and $10 \mathrm{pmol}$ of the following primers 5 L (5'-CCAGATCGCCTTTAAGAAGG-3') and 3 L (5'TACAGGTAGCAGCACGTATG-3'). The amplicon product was $595 \mathrm{bp}$. A nested PCR was then performed with the primers 1XXX (5'-ACTGCACTAGTCCCCCATAC$\left.3^{\prime}\right)$ and $3 \mathrm{~F}^{\prime}$ (5'-ATCGCTGCATAGTCGTAGGC-3'). The product is $251 \mathrm{bp}$. Both HMTV-env PCR reactions were performed in a thermocycler under the following conditions [32] with minimal modifications: a cycle of $95^{\circ} \mathrm{C}$ for $5 \mathrm{~min}$, followed by 35 cycles of $95^{\circ} \mathrm{C}$ for $30 \mathrm{sec}, 58^{\circ} \mathrm{C}$ for $30 \mathrm{sec}, 72^{\circ} \mathrm{C}$ for $30 \mathrm{sec}$, and a final step of $72^{\circ} \mathrm{C}$ for $7 \mathrm{~min}$. Each sample was tested three times to assure the reproducibility of the reaction. In addition, several reactions without template DNA were performed, along with the experimental reactions, to rule out possible contamination of the nuclease-free sterile water and primers.

To identify the amplicon, the gel was transferred onto a positively charged nylon membrane (GE Healthcare Inc., Piscataway, NJ) by capillary action and hybridized with ${ }^{32}$ P-labeled oligonucleotide 1XXXX probe (5'-AGGCCAG GATTTCAAGAACA-3'). The probe was radiolabeled by the end terminal labeling method (New England Biolabs, Ipswich, MA).

After the band was identified by hybridization the remaining PCR product was amplified using the same nested primers and again run in an agarose gel. The nested PCR product (251 bp) was removed from the gel, purified following the conditions recommended by the manufacturer of QIAQuick Gel Extraction Kit (Qiagen 
Inc., Valencia, CA), and submitted for sequencing to the Mount Sinai Genomics Institute Sequencing core facility.

\section{RNA extraction}

RNA was extracted from the $100,000 \times$ g pellet $(\mathrm{P} 2)$ using Trizol LS Reagent following the manufacturer's protocol (Life Technologies, Carlsbad, CA). RNA samples were treated with DNAse and the absence of DNA was confirmed by failing to detect the G3DPH gene sequence by PCR using GE Healthcare Illustra PureTaq Ready To Go PCR Beads, (GE Healthcare, Piscataway NJ), 10 pmol of primers: G3DPH-3 (5'-GGTGAAGACGCCAGTGGACT C-3'), and G3DPH-5 (5'-GTGAAGGTCGGAGTCAACG GA-3') and the conditions previously described [20].

\section{cDNA preparation}

Two sets of cDNA were synthetized using the same P2 RNA samples. One set of cDNA synthesized with oligo$\mathrm{dt}$ was used for the detection of G3DPH and HMTV$e n v$ and the other set with LTR3 primer was used for the detection of HMTV-env/LTR junction sequences.

\section{Oligo-dt CDNA synthesis}

Oligo-dt cDNA was obtained using 2 ug of RNA, oligo-dt primer (Thermo Fisher Scientific, Pittsburgh, PA), and RNaseOut (Life Technologies, Grand Island, NY) following the protocol of the Omniscript Reverse Transcription Kit (Qiagen Inc., Valencia, CA) [20].

\section{Detection of HMTV-env/LTR junction sequences}

CDNA from HMTV-LTR (LTR3 cDNA) was obtained using $2 \mu \mathrm{g}$ of P2 RNA, LTR3 primer (5' -CGAACAGACACAAACACACG-3'), and RNaseOut following the protocol of the Qiagen Omniscript Reverse Transcription Kit [20].

HMTV-env/LTR junction sequences were detected in the LTR3 cDNA by PCR using 10 pmol of primers LTR5MR (5' -ATAAGTCCCTGGTTGCCACC-3') and ENV3LR' (5'-CATATGTGCTGCTACCTGTA-3'), $250 \mathrm{ng}$ of cDNA and the Advantage 2 PCR Kit (Clontech Laboratories, Inc., Mountain View, CA) under the following conditions: $95^{\circ} \mathrm{C}$ for $1 \mathrm{~min}, 35$ cycles of $95^{\circ} \mathrm{C}$ for $30 \mathrm{sec}, 62^{\circ} \mathrm{C}$ for $1 \mathrm{~min}, 68^{\circ} \mathrm{C}$ for $1 \mathrm{~min}$, and finally a cycle of $68^{\circ} \mathrm{C}$ for $10 \mathrm{~min}$. The expected amplicon was $1378 \mathrm{bp}$. A seminested PCR was then performed with the primers ENV3LR' and LTR-1R' (5'-TCAGGAGGAAGGTCGAGT TCT-3') under the following conditions: $95^{\circ} \mathrm{C}$ for $1 \mathrm{~min}$, 35 cycles of $95^{\circ} \mathrm{C}$ for $30 \mathrm{sec}, 62^{\circ} \mathrm{C}$ for $1 \mathrm{~min}, 68^{\circ} \mathrm{C}$ for $1 \mathrm{~min}$, and finally a cycle of $68^{\circ} \mathrm{C}$ for $10 \mathrm{~min}$ [20]. The product was $1018 \mathrm{bp}$. Each sample was tested three times to assure the reproducibility of the reaction. In addition, several reactions without template DNA were performed, along with the experimental reactions, to rule out possible contamination of the nuclease-free sterile water and primers. To identify the amplicon, the gel was then transferred and hybridized with a ${ }^{32}$ P-labeled oligonucleotide env/LTR probe $\left(5^{\prime}\right.$-CTGCAGCAGAAATGGTTGA A-3') [20].

After amplicons were detected on film, the remaining PCR products were re-amplified using the same primers. The amplified PCR product was run in a $1 \%$ agarose gel, isolated and purified using the Quick Gel Extraction Kit and submitted for sequencing at the Mount Sinai Genomics Institute Sequencing facility. The sequences were compared to sequences present in the GenBank. All procedures were carried out in a laminar flow hood based in a laboratory with exclusive use for human tissues.

\section{Detection of murine DNA}

Although the experiments were performed in a room restricted to human tissue work, a series of PCRs were done to detect mouse DNA contamination in any human DNA in which HMTV sequences were detected. Two assays were used to detect murine contaminant DNA [32].

One of these assays is for the detection of cytochrome oxidase, a gene that is part of MoMt [32]. To test for MoMt, the following primers were used: $\mathrm{mt15982 \textrm {F }}$ (5' - AGACGCACCTACGGTGAAGA-3') and mt16267R (5'-AGAGTTTTGGTTCACGGAACATGA-3') [32]. The product yields an amplicon of $286 \mathrm{bp}$. A semi-nested PCR was then performed using the primers mt16115F (5'-TGCCAAACCCCAAAAACACT-3') and mt16267R, which results in a $153 \mathrm{bp}$ amplicon [32]. The PCR product was transferred from the gel to a positively charged nylon membrane and hybridized with a ${ }^{32}$ P-labeled oligonucleotide MoMt DNA probe (5'-GAACTAGAATTGATCAGG CAT-3'). The sensitivity of this reaction has been improved by the hybridization step and is $1.25 \mathrm{fg}$.

The other assay is for the detection of IAP. IAP are retrotransposon elements present at the level of about 1000 copies of varying length per mouse genome. IAP PCR has been proposed as an assay for the detection of murine DNA contamination of human DNA specimens [33]. Amplification of the IAP sequences from the mouse genome by PCR was carried out using the following primers: forward primer ( $5^{\prime}$-ATAATCTGCGCATGAGC CAAGG-3') and reverse primer (5'-AGGAAGAACAC CACAGACCAGA-3) under the recommended conditions [33]: one cycle of $95^{\circ} \mathrm{C}, 5$ minutes, 35 cycles of $95^{\circ} \mathrm{C} 30$ seconds, $58^{\circ} \mathrm{C} 30$ seconds, $72^{\circ} \mathrm{C} 20$ seconds and one cycle of $72^{\circ} \mathrm{C}, 7$ minutes. Products of variable size, reflecting diversity of the IAP, were visualized in a $2 \%$ ethidium bromide stained agarose gel [33]. The sensitivity of this reaction is $1.25 \mathrm{pg}$.

\section{Statistical analysis}

Statistical analysis was performed using a Chi-square test to determine the association of the presence of HMTV sequences in the DNA of milk cells from the women of 
the Biopsy or Reference Group [34]. This test does not introduce multivariate control for other possible differences between Biopsy and Reference Groups. However, other covariates were also examined in bivariate tests (which adjusted for sample clustering differences between the two groups of subjects when necessary) and were not found to be significantly associated with the presence of HMTV sequences.

\section{Abbreviations}

MMTV: Mouse mammary tumor virus; HMTV: Human mammary tumor virus; LTR: Long-Terminal-Repeat; Env: Envelope; PCR: Polymerase chain reaction; RT: Reverse transcriptase; HERV: Human endogenous retrovirus; MoMt: Murine mitochondrial DNA; IAP: Intracisternal-A-type particles; G3PDH: Glycerol-3-phosphate dehydrogenase; HTLV: Human T cell leukemia virus; IRB: Internal review board.

\section{Competing interests}

The authors declare no competing interest.

\section{Authors' contributions}

TN, HM and TM performed the laboratory experiments; KFA was responsible for supplying the milk specimens. DLA carried out the statistical analysis; SMM and BGTP designed the experiments and supervised the experiments. SMM, BGTP, PE and JFH were involved with the writing of manuscript. All authors read and approved the manuscript.

\section{Acknowledgements}

Supported in part by the Avon Foundation (BG-TP, KFA), the TJ Martell Foundation for Leukemia, Cancer, and AIDS Research (TN, TM, HM, SMM, JFH, BG-TP), the Ruttenberg Family Foundation, the Faith Lynn Kash Foundation, and the Myra Shaw Cancer Fund. James F. Holland serves as an uncompensated consultant and as a member of the National Medical Advisory Board of the T.J. Martell Foundation.

\section{Author details}

${ }^{1}$ The Tisch Cancer Institute and Department of Medicine, Icahn School of Medicine at Mount Sinai, 1 Gustave L. Levy Place, New York, NY 10029, USA. ${ }^{2}$ Department of Veterinary and Animal Sciences, University of Massachusetts at Amherst, 240 Thatcher Road, Amherst, MA 01003, USA. ${ }^{3}$ Department of Sociology, Sloan College, University of South Carolina, Rm 321. 911 Pickens St., Columbia, SC 29208, USA. ${ }^{4}$ Department of Microbiology, Icahn School of Medicine at Mount Sinai, 1 Gustave L. Levy Place, New York, NY 10029, USA.

Received: 19 March 2014 Accepted: 12 May 2014

Published: 17 June 2014

\section{References}

1. Feller WF, Chopra HC: Studies of human milk in relation to the possible viral etiology of breast cancer. Cancer 1969, 24(6):1250-1254. PMID: 4311119.

2. Feller WF, Chopra HC: Virus-like particles in human milk. Cancer 1971 , 28(6):1425-1430. PMID: 5001818.

3. Moore DH, Charney J, Kramarsky B, Lasfargues EY, Sarkar NH, Brennan MJ, Burrows JH, Sirsat SM, Paymaster JC, Vaidya AB: Search for a human breast cancer virus. Nature 1971, 229(5287):611-614. PMID: 4925461.

4. Schlom J, Spiegelman S, Moore DH: Detection of high-molecular-weight RNA in particles from human milk. Science 1972, 175(4021):542-544. PMID: 4332822.

5. Dion AS, Farwell DC, Pomenti AA, Girardi AJ: A human protein related to the major envelope protein of murine mammary tumor virus: identification and characterization. Proc Natl Acad Sci USA 1980, 77(3):1301-1305. PMID: 6154929.

6. Litvinov SV, Golovkina TV: Expression of proteins immunologically related to murine mammary tumour virus (MMTV) core proteins in the cells of breast cancer continuous lines MCF-7, T47D, MDA-231 and cells from human milk. Acta Virol 1989, 33(2):137-142. PMID: 2569809.

7. Litvinov SV, Golovkina TV, Kriukova IN, Vasilevskaia LN: A protein related to the main core protein of the mouse mammary cancer virus in a microparticle fraction of human milk. Biull Eksp Biol Med 1987, 103(3):338-340. PMID: 3030463

8. Westley B, May FE: The human genome contains multiple sequences of varying homology to mouse mammary tumour virus DNA. Gene 1984, 28(2):221-227. PMID: 6329918.

9. Glenn WK, Whitaker NJ, Lawson JS: High risk human papillomavirus and Epstein Barr virus in human breast milk. BMC Res Notes 2012, 5:477. PMID: 22937830.

10. Pillay K, Coutsoudis A, York D, Kuhn L, Coovadia HM: Cell-free virus in breast milk of HIV-1- seropositive women. J Acquir Immune Defic Syndr 2000, 24(4):330-336. PMID: 11015149.

11. Johal H, Ford C, Glenn W, Heads J, Lawson J, Rawlinson W: Mouse mammary tumor like virus sequences in breast milk from healthy lactating women. Breast Cancer Res Treat 2011, 129(1):149-155. PMID: 21365265.

12. Wang Y, Holland JF, Bleiweiss IJ, Melana S, Liu X, Pelisson I, Cantarella A, Stellrecht K, Mani S, Pogo BG: Detection of mammary tumor virus env gene-like sequences in human breast cancer. Cancer Res 1995, 55(22):5173-5179. PMID: 7585568.

13. Melana SM, Holland JF, Pogo BG: Search for mouse mammary tumor virus-like env sequences in cancer and normal breast from the same individuals. Clin Cancer Res 2001, 7(2):283-284. PMID: 11234880.

14. Liu B, Wang Y, Melana SM, Pelisson I, Najfeld V, Holland JF, Pogo BG: Identification of a proviral structure in human breast cancer. Cancer Res 2001, 61(4):1754-1759. PMID: 11245493.

15. Etkind P, Du J, Khan A, Pilliteri J, Wiernik PH: Mouse mammary tumor virus-like ENV gene sequences in human breast tumors and in a lymphoma of a breast cancer patient. Clin Cancer Res 2000, 6(4):1273-1278. PMID: 10778951.

16. Ford CE, Tran D, Deng Y, Ta VT, Rawlinson WD, Lawson JS: Mouse mammary tumor virus- like gene sequences in breast tumors of Australian and Vietnamese women. Clin Cancer Res 2003, 9(3):1118-1120. PMID: 12631616.

17. Luo T, Wu XT, Zhang MM, Qian K: Study of mouse mammary tumor virus-like gene sequences expressing in breast tumors of Chinese women. Sichuan Da Xue Xue Bao Yi Xue Ban 2006, 37(6):844-846. 851. PMID: 17236577.

18. Zammarchi F, Pistello M, Piersigilli A, Murr R, Di Cristofano C, Naccarato AG Bevilacqua G: MMTV-like sequences in human breast cancer: a fluorescent PCR/laser microdissection approach. J Pathol 2006, 209(4):436-444. PMID: 16710841.

19. Zapata-Benavides P, Saavedra-Alonso S, Zamora-Avila D, Vargas-Rodarte C, Barrera-Rodríguez R, Salinas-Silva J, Rodríguez-Padilla C, Tamez-Guerra R, Trejo-Avila L: Mouse mammary tumor virus-like gene sequences in breast cancer samples of Mexican women. Intervirology 2007, 50(6):402-407. PMID: 17975321

20. Melana SM, Nepomnaschy I, Sakalian M, Abbott A, Hasa J, Holland JF, Pogo BG: Characterization of viral particles isolated from primary cultures of human breast cancer cells. Cancer Res 2007, 67(18):8960-8965. PMID: 17875739.

21. Melana SM, Nepomnaschy I, Hasa J, Djougarian A, Djougarian A, Holland JF, Pogo BG: Detection of human mammary tumor virus proteins in human breast cancer cells. J Virol Methods 2010, 163(1):157-161. PMID: 19781575.

22. Pogo BG, Holland JF, Levine PH: Human mammary tumor virus in inflammatory breast cancer. Cancer 2010, 116(11 Suppl):2741-2744. PMID: 20503403.

23. Wang Y, Melana SM, Baker B, Bleiweiss I, Fernandez-Cobo M, Mandeli JF, Holland JF, Pogo BG: High prevalence of MMTV-like env gene sequences in gestational breast cancer. Med Oncol 2003, 20(3):233-236. PMID: 14514972.

24. Wong CM, Anderton DL, Smith-Schneider S, Wing MA, Greven MC, Arcaro KF: Quantitative analysis of promoter methylation in exfoliated epithelial cells isolated from breast milk of healthy women. Epigenetics 2010, 5(7):645-655. PMID: 20716965

25. Browne EP, Punska EC, Lenington S, Otis CN, Anderton DL, Arcaro KF: Increased promoter methylation in exfoliated breast epithelial cells in women with a previous breast biopsy. Epigenetics 2011, 6(12):1425-1435. PMID: 22139572

26. National Cancer Institute: www.cancer.gov/cancertopics/factsheet/ detection/probability-breast-cancer.

27. Potischman N, Troisi R: In-utero and early life exposures in relation to risk of breast cancer. Cancer Causes Control 1999, 10(6):561-573. PMID: 10616825 
28. Lawson JS, Heads J, Glenn WK, Whitaker NJ: Breastfeeding, breast milk, and viruses. BMC Womens Health 2007, 7:17. PMID: 17919341.

29. Lawson JS, Glenn WK, Salmons B, Ye Y, Heng B, Moody P, Johal H, Rawlinson WD, Delprado W, Lutze-Mann L, Whitaker NJ: Mouse mammary tumor virus-like sequences in human breast cancer. Cancer Res 2010, 70(9):3576-3585. PMID: 20388779.

30. Hino S: Establishment of the milk-borne transmission as a key factor for the peculiar endemicity of human T-lymphotropic virus type 1 (HTLV-1): the ATL Prevention Program Nagasaki. Proc Jpn Acad Ser B Phys Biol Sci 2011, 87(4):152-166. PMID: 21558754.

31. Resnick RM, Cornelissen MT, Wright DK, Eichinger GH, Fox HS, ter Schegget J, Manos MM: Detection and typing of human papillomavirus in archival cervical cancer specimens by DNA amplification with consensus primers. J Natl Cancer Inst 1990, 82(18):1477-1484. PMID: 2167986.

32. Deligdisch L, Marin T, Lee AT, Etkind P, Holland JF, Melana S, Pogo BG: Human mammary tumor virus (HMTV) in endometrial carcinoma. Int J Gynecol Cancer 2013, 23(8):1423-1428. 23873177.

33. Oakes B, Tai AK, Cingöz O, Henefield MH, Levine S, Coffin JM, Hubert BT: Contamination of human DNA samples with mouse DNA can lead to false detection of XMRV-like sequences. Retrovirology 2010, 7:109. PMID: 21171973.

34. Blair R, Taylor RA: Biostatistics for the Health Sciences. NJ: Pearson Education Inc; 1993.

doi:10.1186/1750-9378-9-20

Cite this article as: Nartey et al:: Human Mammary Tumor Virus (HMTV) sequences in human milk. Infectious Agents and Cancer 2014 9:20.

\section{Submit your next manuscript to BioMed Central and take full advantage of:}

- Convenient online submission

- Thorough peer review

- No space constraints or color figure charges

- Immediate publication on acceptance

- Inclusion in PubMed, CAS, Scopus and Google Scholar

- Research which is freely available for redistribution 